\title{
HIV infection and cancer in the era of highly active antiretroviral therapy (Review)
}

\author{
GIUSEPPE BARBARO $^{1}$ and GIORGIO BARBARINI ${ }^{2}$ \\ ${ }^{1}$ Department of Medical Pathophysiology, University 'La Sapienza’, Rome; \\ ${ }^{2}$ Department of Infectious and Tropical Diseases, IRCCS, San Matteo University of Pavia, Italy
}

Received December 5, 2006; Accepted January 15, 2007

\begin{abstract}
The majority of cancers affecting HIV-infected subjects are those established as acquired immunodeficiency syndrome (AIDS)-defining: Kaposi's sarcoma (KS), nonHodgkin's lymphoma (NHL), and invasive cervical cancer (ICC). However, other types of cancer, such as Hodgkin's disease (HD), anal cancer, lung cancer and testicular germ cell tumors appear to be more common among HIV-infected subjects compared to the general population. While not classified as AIDS-defining, these malignancies have been referred to as AIDS-associated malignancies. The mechanisms by which depressed immunity could increase the risk for cancer are unclear, except for in KS and most subtypes of NHL, where it is strictly associated with a low CD4 count. Although it remains unclear whether HIV-1 acts directly as an oncogenic agent, it may contribute to the development of malignancies through several mechanisms (e.g., infection by oncogenic viruses, impaired immune surveillance, imbalance between cellular proliferation and differentiation). Studies of the effect of highly active antiretroviral therapy (HAART) on the incidence and progression of HIV/AIDS-associated cancers provided contrasting data. While a significant decrease in the incidence of KS has been observed, HAART has not had a significant impact on NHL incidence, particularly systemic NHL, or on ICC, HD, anal cancers and other non-AIDSdefining cancers. Regardless of whether these cancers are directly related to HIV-induced immunodeficiency, treating cancer in HIV-infected patients remains a challenge because of drug interactions, compounded side effects, and the potential effect of chemotherapy on CD4 count and HIV-1 viral load. A better knowledge of viral mechanisms of immune evasion and manipulation will provide the basis for a better management and treatment of the malignancies associated with chronic viral infections.
\end{abstract}

Correspondence to: Dr Giuseppe Barbaro, Viale Anicio Gallo 63, 00174 Rome, Italy

E-mail: g.barbaro@tin.it

Key words: human immunodeficiency virus, acquired immunodeficiency syndrome, cancer, highly active antiretroviral therapy

\section{Contents}

1. Introduction

2. AIDS-defining cancers

3. Non-AIDS-defining cancers

4. Other malignancies in the HIV-infected community

5. Potential causes of cancer in HIV/AIDS

6. The impact of HAART

\section{Introduction}

Cancer is a significant cause of morbidity and mortality in human immunodeficiency virus (HIV)-infected subjects (1). The majority of cancers affecting HIV-infected subjects are those established as acquired immunodeficiency syndrome (AIDS)-defining: Kaposi's sarcoma, non-Hodgkin's lymphoma, and invasive cervical cancer. However, other types of cancer also appear to be more common among HIV-infected subjects compared to the general population. While not classified as AIDS-defining, these malignancies are affecting the HIVinfected community greatly and have been referred to as AIDS-associated malignancies (1). The clinical classification of cancers observed in the HIV-infected community is reported in Table I.

The introduction of highly active antiretroviral therapy (HAART) has resulted in decreased mortality and morbidity, improving the quality of life of HIV-infected subjects. However, the widespread use of HAART altered the incidence of cancer or perhaps even increased the prevalence of some types of cancer in the HIV-infected population providing a new intriguing clinical scenery (1).

\section{AIDS-defining cancers}

Kaposi's sarcoma. In the general population, Kaposi's sarcoma $(\mathrm{KS})$ is a rare, typically indolent cancer that affects older people or those receiving immunosuppressants following an organ transplant. People infected with HIV-1 are 100 to 300 times more likely to develop KS (1-6).

In 1981 the first HIV-associated KS was described in the United States where $35 \%$ of AIDS patients were found to present with this rare tumour. It is common in homosexuals with AIDS but rare in other risk groups. The cause of AIDSassociated KS is presently unknown and causes may be 
Table I. AIDS-associated cancers: clinical classification (1).

AIDS-defining cancers
Kaposi's sarcoma
Non-Hodgkin's lymphoma
Invasive cervical cancer
Non AIDS-defining cancers
Hodgkin's lymphoma
Anal cancer
Lung cancer
Testicular germ tumors
Other cancers in the HIV-infected community
Skin/lip/tongue cancer
Pharynx/larynx cancer
Penis cancer
Liver cancer
Kidney cancer
Pancreas cancer
Gastrointestinal cancers
Brain and central nervous system cancer
Prostate cancer
Bladder cancer
Vulva/vagina cancer
Breast cancer
Leukemia
Multiple myeloma
Soft tissue sarcomas
Leiomyosarcoma
Angiosarcoma
Pand

multiple $(5,7)$. AIDS-associated KS is associated with human herpesvirus-8 (HHV-8), also known as KS-associated herpes virus), although there is no definitive evidence that HHV-8 causes KS (5). The risk and severity of KS increase in the presence of low CD4 count (6) and subjects with an intact immune system tend not to develop $\mathrm{KS}$ when infected with HHV-8 (7).

Studies have unequivocally demonstrated a significant decline in the incidence of KS following the introduction of HAART (5,8-10). In the early 1980 's, KS was the AIDSdefining illness in approximately $30 \%$ of infected individuals; a value which later dropped to $10-15 \%$ in the late 1990s (7). The incidence rates for KS are 5 times lower in HIV-infected patients who have received HAART, compared to those who have not (11). In San Francisco, deaths from KS significantly decreased from $15.6 \%$ of total AIDS deaths in 1994 to $7.1 \%$ in 1998 (12). HAART regimens containing protease inhibitors and non-nucleoside reverse transcriptase inhibitors are equally protective against developing KS (10). Most patients who develop KS while taking HAART show evidence of virologic treatment failure. Importantly, HAART may also have a positive effect on treating established KS, especially in patients without visceral disease (10).
In developing countries where the availability of HAART is scanty an increased incidence of KS has been observed (9). Currently, KS represents one of the most common diseases in the HIV-infected community in Africa with a related high mortality rate for pulmonary involvement (9).

Non-Hodgkin's lymphoma. Compared to the general population the risk of developing a non-Hodgkin's lymphoma (NHL, also referred to as AIDS-associated lymphoma) is significantly increased in the HIV-infected population (from 40 to 400 times, median 150-220 times), depending on the specific study and the type of NHL $(1-6,13,14)$.

NHL encompasses several types of lymphoma, including systemic NHL, primary central nervous system NHL (PCNSL, also referred to as primary brain lymphoma or cerebral lymphoma), and primary effusion lymphoma (PEL) or body cavity-based lymphoma, a rare and aggressive form of NHL $(7,13,15)$. Data also indicate that high-grade lymphoma is more prevalent in the HIV-infected community compared to low-grade lymphoma $(7,13,15)$. The risk of developing NHL, particularly PCNSL, increases with lower CD4 counts, and further progression of HIV infection $(6,7)$.

No definitive conclusions can be drawn regarding the effect of HAART on the incidence of NHL $(1,16)$. Some studies have demonstrated significant decreases in the incidence of NHL following the introduction of HAART, whereby incidence rates decreased by almost 50\% (9), and patients receiving HAART experienced a 5-fold decrease in the incidence of NHL compared to treatment-naive patients (11). However, other studies failed to show any significant change, and even suggested modest increases in the incidence of NHL in HIV-infected subjects $(12,13)$. Significant decreases have been reported for immunoblastic lymphoma in some studies (9), but not in others (8). Incidence of AIDS-associated Burkitt's lymphoma has not decreased and one analysis even demonstrated an increase in incidence after the introduction of HAART, but this increase was not statistically significant (9). Studies showed that the incidence of PCNSL decreased considerably following the introduction of $\operatorname{HAART}(9,11,13)$. Administration of HAART has also been associated with longer survival in patients suffering from PCNSL (17). The effect of HAART on the incidence of PEL is unknown because of the rarity of the disease (1). Interestingly, patients with systemic NHL who received and responded to HAART were significantly more likely to achieve a complete response, suggesting that a patient's response to HAART may provide insight into their cancer prognosis $(14,16,18)$.

Invasive cervical cancer (ICC). HIV-infected women are approximately 5 to 9 times more likely to have ICC compared to HIV-uninfected women $(1,3,6,19)$ and this cancer accounts for $55 \%$ of AIDS-associated malignancies in some settings (20). Human papillomavirus (HPV) is involved in almost all cases of cervical cancer, regardless of HIV status, and is strongly associated with cervical intraepithelial neoplasia (CIN) and squamous intraepithelial lesions (SIL), which are precursors to $\operatorname{ICC}(21,22)$. While the relationship between HIV-1 infection and ICC is not definitive, this is not the case for CIN and SIL (1). In contrast to HIV-uninfected women whose low-grade lesions typically resolve without treatment 
(23), lesions are more likely to progress and to recur after treatment in HIV-infected women in relation to the state of immunodeficiency $(22,24)$.

Several studies have assessed the impact of HAART on the incidence and progression of precancerous cervical lesions. One large study reported no significant changes in the incidence of ICC when rates were compared during the pre-HAART and post-HAART eras (9). Some studies have shown decreased cytologic progression of these lesions $(25,26)$, while others have reported increased progression or non-conclusive results (21). Moreover, HAART was not associated with decreased prevalence or persistence of HPV infection, but a significant reduction in the incidence of HPV-16 and HPV-18 (oncogenic types of HPV) was detected in HAART-treated women, suggesting that HAART may have an effect on acute HPV infection, but not on advanced infection $(1,27)$.

\section{Non-AIDS-defining cancers}

Hodgkin's disease. Although Hodgkin's disease (HD) is not considered an AIDS-defining cancer, with contrasting opinions from some researchers (3), HIV-infected subjects are 7.6 to 11.5 times more likely to have HD compared to the general population $(1-4,6,13,28)$. While analyses have routinely demonstrated an increased risk of HD in HIV-infected subjects, a causal link between HIV-1 and HD has not been established and studies assessing the effect of immunosuppression on the incidence of HD are conflicting $(1,3,4,6,28)$.

Few studies have looked at the effect of HAART on the incidence of HD in HIV disease (1). However, those that have assessed this relationship reported no difference in rates either when comparing patients who had received HAART with treatment-naive patients (29) or when comparing HD rates during the pre-HAART and post-HAART eras (9).

Anal cancer. HIV-infected subjects are 30 to 50 times more likely to have anal cancer, with rates as high as 60 -fold in HIV-infected men who are bisexual or homosexual $(1,30)$. Anal cancer in HIV-infected subjects is strongly associated with HPV infection (specifically, HPV-16 and HPV-18 in high-grade form) (31) and the presence of precancerous anal lesions, which are referred to as squamous intraepithelial lesions (SIL) and anal intraepithelial neoplasia (AIN) $(1,24,32)$.

Until recently, anal sex was assumed to be the mode of acquisition for anal HPV infection. However, when rates of HPV infection and SILs were compared in HIV-infected men with or without a history of bisexual or homosexual behavior, rates were high even in those men with no history of anal sex who contracted HIV infection through intravenous drug use, suggesting that HPV infection may be acquired through means other than anal intercourse $(1,30)$. Moreover, according to a report by Pakefsky et al $76 \%$ of HIV-infected women and $42 \%$ of high-risk HIV-uninfected women tested positive for anal HPV-DNA (33). Presently, it is unclear whether prevalent risk factors, such as anal intercourse, a history of sexually transmitted diseases, or even heavy tobacco use are responsible for the increased incidence of anal cancer in the HIV-infected population (1). It is possible that HIV-induced immunosuppression may facilitate the development of anal cancer in subjects coinfected by HPV, since high-grade and progressive forms of anal cancer have been described in HIV-infected subjects with a low CD4 count (32). However, one study revealed that the incidence was significantly increased even during early HIV infection, suggesting that severe immunosuppression is not necessary for the development of anal cancer in HIV-infected subjects (28).

The possible benefits of HAART on the incidence of anal cancer and anal precancerous lesions have not been conclusively demonstrated (1). Few studies have examined this relationship suggesting that HAART has not decreased the incidence or increased the regression of these lesions $(24,32,34)$. When rates during the pre-HAART and postHAART eras were compared, no significant change in the incidence of anal cancer was observed $(24,34)$.

Lung cancer. HIV-infected subjects are 2.5 to 7.5 times more likely to develop lung cancer compared to the general population $(1-4,6)$. In several studies lung cancer was the most frequently observed non-AIDS-defining malignancy $(6,12,19)$. Several studies have reported a positive correlation between rates of lung cancer and HIV-induced immunosuppression $(3,4)$. Analyses of risk behavior have reported conflicting data: one study showed that HIV-infected patients with lung cancer smoked twice as many cigarettes as HIV-uninfected patients with lung cancer (35), while another study that compared HIV-infected to HIV-uninfected women with similar smoking histories showed a 2-fold increased incidence in the HIV-infected women (19). Long-term cigarette exposure is typically lower in HIV-infected subjects because they are usually diagnosed with lung cancer at an earlier age (36).

Before the introduction of HAART, rates of lung cancer were low, perhaps on account of early AIDS-related mortality (1). A recent analysis showed an almost 9-fold increase in lung cancer incidence following the introduction of HAART (36). Unfortunately, the outcome of these patients remains poor despite HAART (1).

Testicular germ cell tumors. HIV-infected men are 1.4 to 8.2 times more likely to develop testicular cancer compared to the general population $(2-4,6,31,37)$, although other studies failed to show a significantly increased incidence (28). While no viral oncogene has been implicated in HIV-associated testicular cancer, viruses such as mumps orchitis, HPV, Epstein-Barr virus, and human endogenous retrovirus K10 are associated with testicular cancer in HIV-uninfected men and may be involved in development of testicular cancer also in HIV-infected subjects $(37,38)$, independently of the state of immunodeficiency $(3,37)$.

The effect of HAART on incidence rates has not been analyzed thoroughly, but one report showed no difference in incidence rates when comparing the pre-HAART and postHAART eras (37).

\section{Other malignancies in the HIV-infected community}

In addition to the cancers discussed above, the risk of developing several other cancers appears to be slightly increased in HIV-infected subjects: leukemia $(3,4,28)$, pharynx $(3,4)$, pancreas $(3)$, multiple myeloma $(2-4,28)$, esophagus $(3,4)$, 


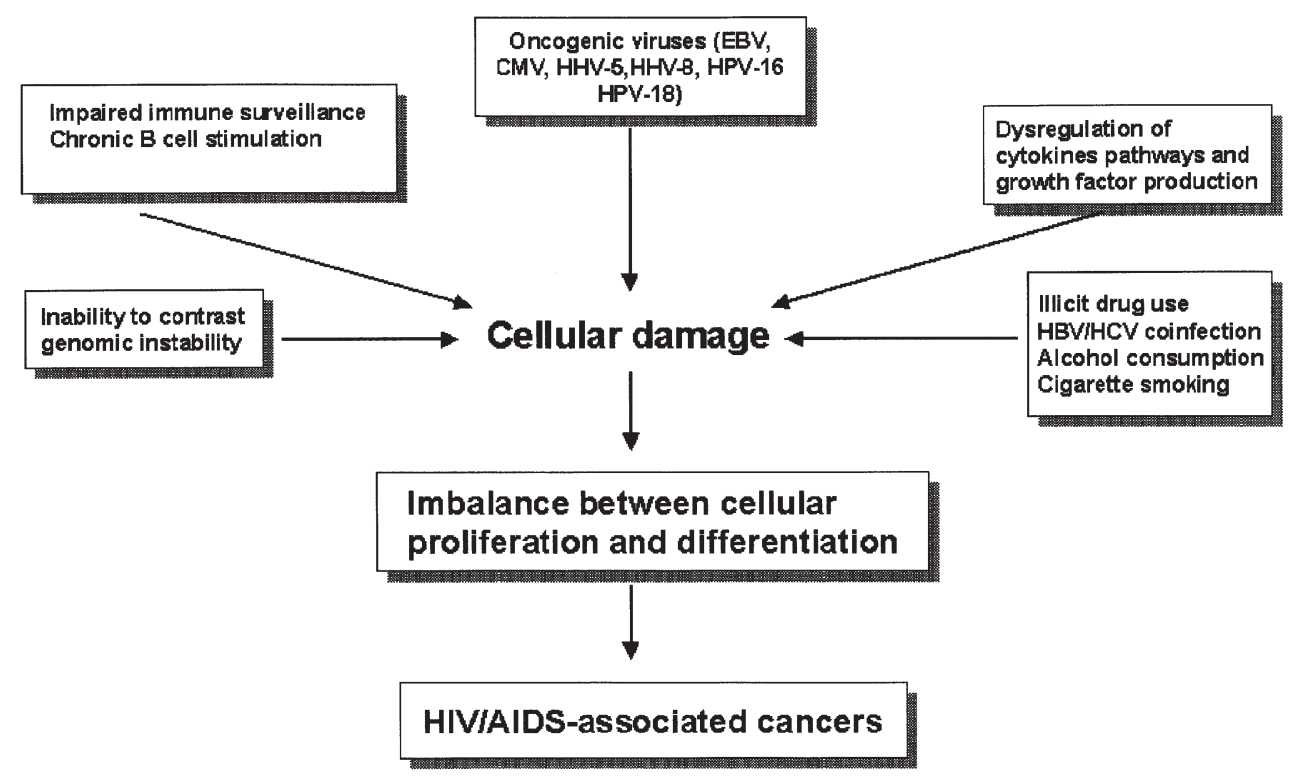

Figure 1. Potential mechanisms implicated in the development of cancer in HIV/AIDS. EBV, Epstein-Barr virus; CMV, cytomegalovirus; HHV, human herpes virus; HPV, human papillomavirus; $\mathrm{HBV}$, hepatitis B virus; $\mathrm{HCV}$, hepatitis $\mathrm{C}$ virus.

liver $(3,8,28)$, skin $(2-4,31)$, lip $(3,28)$, kidney (3), penile (3), tongue (4), colorectal (4), vulva/vagina (3), stomach $(3,4)$, brain and central nervous system (2-4), leiomyosarcoma $(3,31)$, larynx $(3,4)$, heart $(3)$, and angiosarcoma (2). The incidence of certain types of cancers, such as prostate $(4,4,6)$, breast $(3,6)$ and bladder cancer $(4)$ appears to be decreased in the HIV-infected community, especially after the introduction of HAART (1), although a small case study reported a nonsignificant increase in the incidence of prostate cancer in HIV-infected men (39). The rates of breast cancer in men may have increased to some extent, especially in intravenous drug users (3). Further controlled clinical and epidemiological studies are needed to better define the trend of these HIVassociated malignancies after the introduction of HAART.

\section{Potential causes of cancer in HIV/AIDS}

Although it remains unclear whether HIV-1 acts directly as an oncogenic agent, it may contribute to the development of malignancies through several mechanisms (Fig. 1).

Infection by oncogenic viruses (e.g., cytomegalovirus, Epstein-Barr virus, HHV-5, HHV-8, HPV-16 and HPV-18), impaired immune surveillance, dysregulation of cytokine pathways and growth factor production, chronic B cell stimulation, and imbalance between cellular proliferation and differentiation may all contribute to the development of HIV/ AIDS-associated malignancies $(1,5-7,12,13)$. Uncontrolled viral infection may play a significant causative role in many HIV/AIDS-associated cancers according to the immunodeficiency state of the subject (1).

Many HIV-associated malignancies affect sites that are in contact with the outside environment (e.g., cervix, lung, oral cavity, skin, and anus). The increased density of immune cells and coincident elevated concentration of HIV-1 at these sites could lead to local compromised immune defenses and the subsequent development of malignancies at these sites $(1,40)$. Alternatively, risk factors present in the HIV-infected community, including multiple sexual partners, hepatitis B virus/hepatitis $C$ virus coinfection, illicit drug use, increased alcohol consumption and cigarette smoking, could account for the increased rates of these cancers (1). For example, compared to HIV-uninfected patients with cancer, more HIV-infected patients with cancer have a history of cigarette smoking and illicit drug use $(35,40)$.

\section{The impact of HAART}

Although it may be premature to draw any definitive conclusions, preliminary data suggest that with the exception of $\mathrm{KS}$, HAART has not had a significant impact on cancer incidence in the HIV-infected population (1). Widespread availability of HAART has only occurred within the last decade and many of the malignancies discussed above require several years to develop. Because of HAART's effect on the incidence of KS (5,8-10), one would expect malignancies that are associated with immunosuppression to undergo a significant decline in the incidence following the widespread availability of HAART, especially in developed countries. If immunodeficiency is a key factor in the development of these tumors, the improvement of immunologic functions associated with HAART would slow tumor progression. Unfortunately, this has not been the case (1). For example, evidence suggests that NHL occurs more frequently in immunocompromised patients $(6,8,15)$, but HAART has not had a significant impact on NHL incidence, particularly systemic NHL $(8,9,11-13,16)$.

It has been speculated that the extended survival afforded by HAART, in conjunction with incomplete immune restoration, may increase the incidence of some cancers (1). Prolonged exposure to viral oncogenes, moderate immune suppression, and genomic instability could result in impaired immune surveillance and the subsequent development of tumors $(1,32,36,37)$. Based on this scenery, the incidence of tumors associated with chronic moderate immune suppression would be expected to increase. In fact, the incidence of lung 
cancer appears to increase after the introduction of HAART $(1,36)$. Another explanation could be that prior to the introduction of HAART, patients typically died of opportunistic infections or other HIV-associated complications prior to developing a malignancy, some of which take years to develop (1). It is unclear whether HAART will ever provide full immune recovery in $\mathrm{HIV}$-infected subjects, a situation that may be necessary in order to decrease cancer incidence as a whole in this specific population (1). Some researchers speculate that cancer rates, specifically of AIDS-associated lymphoma, will rise in areas with widespread availability to HAART (41), though others disagree (9).

Regardless of whether these cancers are directly related to HIV-induced immunodeficiency, treating cancer in HIVpositive patients remains a challenge because of drug interactions, compounded side effects, and the potential effect of chemotherapy on CD4 count and HIV-1 viral load $(1,37,38,42)$. Moreover, treatment compliance by HIV-infected patients with cancer may be poor, possibly because of the increased responsibility of taking drugs for both diseases with associated increased rate of compounded side effects (1). The question of whether to suspend HAART during chemotherapy depends on several factors, particularly the type and stage of malignancy and the stage of HIV infection $(16,43,44)$. A better knowledge of viral mechanisms of immune evasion and manipulation will provide the basis for a better management and treatment of the malignancies associated with chronic viral infections.

\section{References}

1. Newcomb-Fernandez J: Cancer in the HIV-infected population. Res Initiat Treat Action 9: 5-13, 2003.

2. Goedert JJ, Cotè TR, Virgo P, et al: Spectrum of AIDS-associated malignant disorders. Lancet 351: 1833-1839, 1998.

3. Frisch M, Biggar RJ, Engels EA, Goedert JJ for the AIDSCancer Match Registry Study Group. Association of cancer with AIDS-related immunosuppression in adults. JAMA 285: 1736-1745, 2001.

4. Gallagher B, Wang Z, Schymura MJ, Kahn A and Fordyce EJ: Cancer incidence in New York State Acquired Immunodeficiency Syndrome patients. Am J Epidemiol 154: 544-556, 2001.

5. Spano JP, Atlan D, Breau JL and Farge D: AIDS and non-AIDSrelated malignancies: a new vexing challenge in HIV-positive patients. Part I: Kaposi's sarcoma, and Hodgkin's lymphoma. Eur J Int Med 13: 170-179, 2002.

6. Mbulaiteye SM, Biggar RJ, Goedert JJ and Engels EA: Immune deficiency and risk for malignancy among persons with AIDS. J Acquir Immune Defic Syndr 32: 527-533, 2003.

7. Gates AE and Kaplan LD: AIDS malignancies in the era of highly active antiretroviral therapy. Oncology 16: 441-459, 2002.

8. Rabkin CS: AIDS and cancer in the era of highly active antiretroviral therapy (HAART). Eur J Cancer 37: 1316-1319, 2001.

9. International Collaboration on HIV and Cancer: Highly active antiretroviral therapy and incidence of cancer in human immunodeficiency virus-infected adults. J Natl Cancer Inst 92: 1823-1830, 2000

10. Portsmouth S, Stebbing J, Gill J, et al: A comparison of regimens based on non-nucleoside reverse transcriptase inhibitors or protease inhibitors in preventing Kaposi's sarcoma. AIDS 17: F17-F22, 2003.

11. Carrieri MP, Pradier C, Piselli P, et al: Reduced incidence of Kaposi's sarcoma and of systemic non-Hodgkin's lymphoma in HIV-infected individuals treated with highly active antiretroviral therapy. Int J Cancer 103: 142-144, 2002.

12. Louie JK, Hsu LC, Osmond DH, Katz MH and Schwarcz SK: Trends in causes of death among persons with Acquired Immunodeficiency Syndrome in the era of highly active antiretroviral therapy, San Francisco, 1994-1998. J Infect Dis 186: 1023-1027, 2002.
13. Dal Maso L and Franceschi S: Epidemiology of non-Hodgkin lymphomas and other haemolymphopoietic neoplasms in people with AIDS. Lancet Oncol 4: 110-119, 2003.

14. Hoffmann C, Wolf E, Fatkenheuer G, et al: Response to highly active antiretroviral therapy strongly predicts outcome in patients with AIDS-related lymphoma. AIDS 17: 1521-1529, 2003.

15. Gates AE and Kaplan LD: AIDS malignancies in the era of highly active antiretroviral therapy. Oncology 16: 657-665, 2002.

16. Clayton A and Mughal T: The changing face of HIV-associated lymphoma: what can we learn about optimal therapy in the post highly active antiretroviral therapy era? Hematol Oncol 22: 111-120, 2004.

17. Skiest DJ and Crosby C: Survival is prolonged by highly active antiretroviral therapy in AIDS patients with primary nervous system lymphoma. AIDS 17: 1787-1793, 2003.

18. Hoffmann C, Chow KU, Wolf E, et al: Strong impact of highly active antiretroviral therapy on survival in patients with human immunodeficiency virus-associated Hodgkin's disease. Br J Haematol 125: 455-462, 2004

19. Phelps RM, Smith DK, Heilig CM, et al. Cancer incidence in women with or at risk for HIV. Int J Cancer 94: 753-757, 2001.

20. Maiman M, Fruchter RG, Clark M, Arrastia CD, Matthews R and Gates EJ: Cervical cancer as an AIDS defining illness. Obstet Gynecol 89: 76-80, 1997.

21. Delmas MC, Larsen C, van Benthem B, et al: Cervical squamous intraepithelial lesions in HIV-infected women: prevalence, incidence and regression. European Study Group on Natural History of HIV Infection in Women. AIDS 14: 1775-1784, 2000.

22. Clarke B and Chetty R: Postmodern cancer: the role of human immunodeficiency virus in uterine cervical cancer. Mol Pathol 55: 19-24, 2002.

23. Robinson WR, Luck MB, Kendall MA and Darragh TM: The predictive value of cytologic testing in women with the human immunodeficiency virus who have low-grade squamous cervical lesions: a substudy of a randomized, phase III chemoprevention trial. Am J Obstet Gynecol 188: 896-900, 2003.

24. de Sanjosè S and Palefsky J: Cervical and anal HPV infection in HIV positive women and men. Virus Res 89: 210-211, 2002.

25. Minkoff H, Ahdieh L, Massad LS, et al: The effect of highly active antiretroviral therapy on cervical cytologic changes associated with oncogenic HPV among HIV-infected women. AIDS 15: 2157-2164, 2001.

26. Heard I, Tassie JM, Kazatchkine MD and Orth G: Highly active antiretroviral therapy enhances regression of cervical intraepithelial neoplasia in HIV-seropositive women. AIDS 16: 1799-1802, 2002.

27. Lillo FB, Ferrari D, Veglia F, et al: Human papillomavirus infection and associated cervical disease in human immunodeficiency virus-infected women: effect of highly active antiretroviral therapy. J Infect Dis 184: 547-551, 2001.

28. Grulich AE, Li Y, McDonald A, Correll PKL, Law MG and Kaldor JM: Rates of non-AIDS-defining cancers in people with HIV infection before and after AIDS diagnosis. AIDS 16: 1155-1161, 2002.

29. Vilchez RA, Finch CJ, Jorgensen JL and Butel JS: The clinical epidemiology of Hodgkin lymphoma in HIV infected patients in the highly active antiretroviral therapy (HAART) era. Medicine 82: 77-81, 2003

30. Piketty C, Darragh TM, Da Costa M, et al: High prevalence of anal human Papillomavirus infection and anal cancer precursors among HIV-infected persons in the absence of anal intercourse. Ann Intern Med 138: 453-459, 2003.

31. Spano JP, Atlan D, Breau JL and Farge D: AIDS and non-AIDS related malignancies: a new vexing challenge in HIV-positive patients. Part II. Cervical and anal squamous epithelial lesions, lung cancer, testicular germ cell cancers and skin cancers. Eur J Int Med 13: 227-232, 2002.

32. Palefsky J: Anal squamous intraepithelial lesions: relation to HIV and human papillomavirus infection. J Acquir Immune Defic Syndr 21 (suppl 1): S42-S48, 1999.

33. Palefsky JM, Holly EA, Ralston ML, Da Costa M and Greenblatt RM: Prevalence and risk factors for anal Papillomavirus infection in human immunodeficiency virus (HIV)-positive and high-risk HIV-negative women. J Infect Dis 183: 383-391, 2001.

34. Bower M, Powles T, Newsom-Davis T, et al: HIV-associated anal cancer: has highly active antiretroviral therapy reduced the incidence or improved the outcome? J Acquir Immune Defic Syndr 37: 1563-1565, 2004. 
35. Tirelli U, Spina M, Sandri S, et al: Lung carcinoma in 36 patients with human immunodeficiency virus infection. Cancer 88: 563-569, 2000

36. Bower M, Powles T, Nelson M, et al: HIV-related lung cancer in the era of highly active antiretroviral therapy. AIDS 17: 371-375, 2003.

37. Powles T, Bower M, Daugaard G, et al: Multicenter study of human immunodeficiency virus-related germ cell tumors. J Clin Oncol 21: 1922-1927, 2003.

38. Fizazi K, Amato RJ, Beuzeboc P, et al: Germ cell tumors in patients infected by the human immunodeficiency virus. Cancer 92: 1460-1467, 2001.

39. Crum N, Hale U, Utz G and Wallace M: Increased risk of prostate cancer in HIV infection? AIDS 16: 1703-1704, 2002.

40. Demopoulos BP, Vamvakas E, Ehrlich JE and Demopoulos R: Non-Acquired Immunodeficiency Syndrome-defining malignancies in patients infected with Human Immunodeficiency Virus. Arch Path Lab Med 127: 589-592, 2003.
41. Clarke CA and Glaser SL: Epidemiologic trends in HIVassociated lymphomas. Curr Opin Oncol 13: 354-359, 2001.

42. El-Rayes BF, Berenij K, Schuman P and Philip PA: Breast cancer in women with human immunodeficiency virus infection: implications for diagnosis and therapy. Breast Cancer Res Treat 76: 111-116, 2002.

43. Ratner L, Lee J, Tang S, et al: Chemotherapy for human immunodeficiency virus-associated non-Hodgkin's lymphoma in combination with highly active antiretroviral therapy. J Clin Oncol 19: 2171-2178, 2001.

44. Little RF, Pittaluga S, Grant N, et al: Highly effective treatment of acquired immunodeficiency syndrome-related lymphoma with dose-adjusted EPOCH: impact of antiretroviral therapy suspension and tumor biology. Blood 101: 4653-4659, 2003. 